\title{
Malignant Melanoma Metastasis to the Appendix as the First Presentation of Lentigo Maligna Melanoma
}

\author{
Shokouh Taghipour Zahir, MD, AP, $\mathrm{CP}^{1 *}$; Koorosh Rahmani, MD ${ }^{1}$; Seyed Abolfazl Hosseini, MD \\ 'Shahid Sadoughi General Hospital, Shahid Sadoughi University of Medical Sciences, Yazd, Iran
}

\begin{abstract}
Malignant melanoma is a malignant neoplasm of the skin and mucosal tissues, and its behavior is not predictable. Thus, it could metastasize via mysterious routes. Here, we report a rare case of acute abdomen and acute appendicitis which involved metastatic malignant melanoma in a 63-year-old man without a history of previously treated malignant melanoma.

Keywords: Appendicitis, Malignant melanoma, Metastasis

Cite this article as: Taghipour Zahir S, Rahmani K, Hosseini SA. Malignant melanoma metastasis to the appendix as the first presentation of lentigo maligna melanoma. Arch Iran Med. 2021;24(4):330-332. doi: 10.34172/aim.2021.46
\end{abstract}

Received: November 14, 2020, Accepted: December 23, 2020, ePublished: April 1, 2021

\section{Introduction}

Malignant melanoma is a type of skin neoplasm that originates from neoplastic transformation of melanocytes, which are located in the skin and mucosal tissues. Exposure to ultraviolet light (UV) seems to be the most important initiator of malignant transformation of the melanocytes. However, other factors, such as genetic predisposition, can make a person more prone to malignant melanoma. ${ }^{1,2}$ Malignant melanoma has variable histological subtypes such as superficial spreading malignant melanoma, nodular malignant melanoma, lentigo maligna melanoma, Hutchinson freckle, and acral lentiginous malignant melanoma. ${ }^{1}$ The most common sites of involvement are the skin of the head and neck, lower extremities, and back. Other areas that could be affected are the oral cavity, anogenital mucosa, esophagus, leptomeningeal membrane, and also the eyes. ${ }^{2,3}$ The prognosis of malignant melanoma depends on various factors such as high Breslow thickness involvement in the primary tumor, high Clark level, lymph vascular invasion, male gender, neurotropism, surface ulceration, and intra-tumoral lymphocytic infiltration. ${ }^{4,5}$ Metastasis to the peritoneum, liver, regional, and nonregional lymph nodes, lungs, and brain is common., Metastasis to the appendix is rare, and rarer still is metastasis to the appendix as the first presentation without any previous history of malignant melanoma. Here, we report a rare case of metastatic malignant melanoma to the appendix as the first presentation of lentigo maligna melanoma.

\section{Case Report}

A 63-year-old man was admitted to the emergency department with symptoms of an acute abdomen. Lab tests showed leukocytosis $\left(15 \times 10^{3} / \mu \mathrm{L}\right)$ with increased erythrocyte sedimentation rate. On physical examination, he had right lower quadrant pain with nausea and vomiting. Acute appendicitis was considered for him and he underwent laparoscopic appendectomy. The received specimen consisted of appendix M $8 \times 1 \mathrm{~cm}$ covered by a fibrinopurulent exudate. On section, the lumen was filled by pus and was dilated. On microscopic examination, the entire wall was severely infiltrated by neutrophils with spread of inflammatory cells into the peri-appendiceal fatty tissue. The wall was also involved by neoplastic cells with round to oval shaped nuclei without conspicuous nucleoli and scanty eosinophilic cytoplasm. Neoplastic cells were arranged in single cells, solid nests, and pseudoglandular patterns. The peri-appendiceal fatty tissue was also involved by neoplastic cells. Focally brown pigment was deposited in the cytoplasm of neoplastic cells (Figure 1). In differential diagnosis, neuroendocrine tumors, metastatic carcinoma, and malignant melanoma were considered. An immunohistochemistry (IHC) panel composed of AE1/AE3, CK7, CK20, chromogranin A, NSE, synaptophysin, and HMB-45 was selected for differential diagnosis. The neoplastic cells had a negative reaction pattern for AE1/AE3, CK7, CK20, NSE, chromogranin A and synaptophysin, and had a positive reaction for HMB-45 and S-100 (Figure 2). Based on IHC studies, metastatic malignant melanoma was confirmed. The patient's hospital chart was reviewed for the possible previous primary origin of malignant melanoma, but the patient did not have any document for it. For clinical examination, the patient was called to the pathology department. During the examination, a black plaque-like spot M $1.5 \mathrm{~cm}$ in diameter was observed on the skin of the temporal area of the face, which had an irregular margin. No ulceration or satellite nodules were 


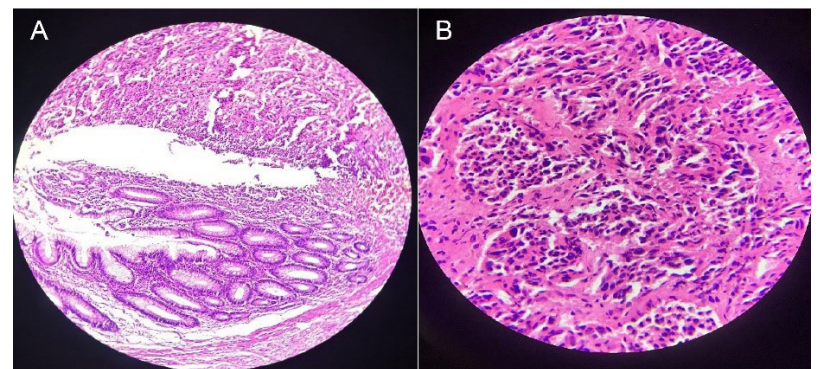

Figure 1. (A) Appendix wall involved by neoplastic cells that have dark oval shape nuclei. (B) Melanin pigment in the cytoplasm of neoplastic cells (H\&E staining, $\times 20$ ).

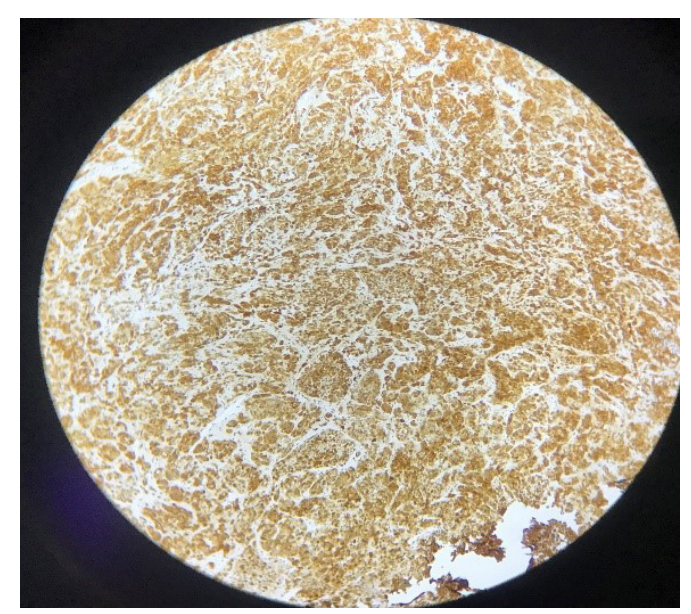

Figure 2. Positive Reaction of Neoplastic Cells for HMB-45 (IHC staining, $\times 20$ ).

observed (Figure 3). The patient stated that the lesion had developed about two years ago. As a primary source of malignant melanoma, the lesion was surgically removed with sufficient margins. On microscopic examination, neoplastic melanocytic cells with hyperchromatic nuclei

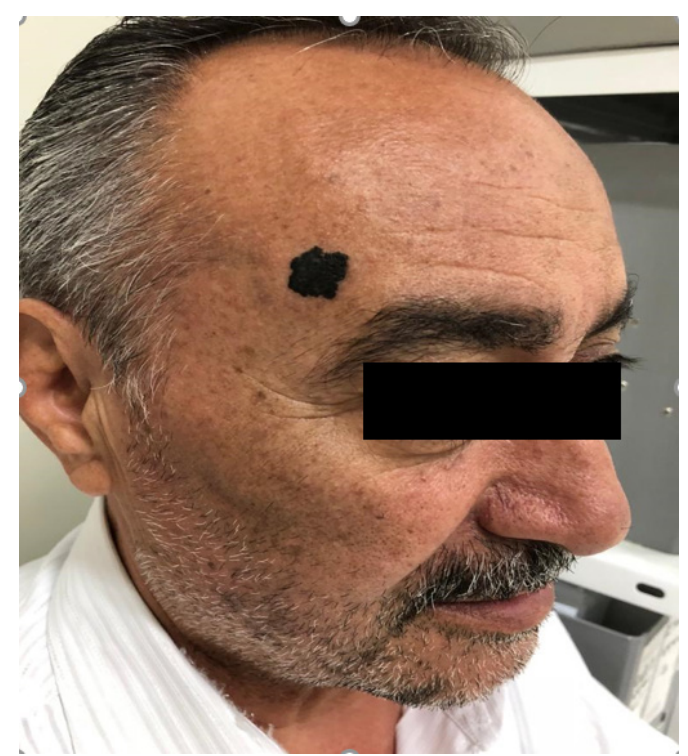

Figure 3. Raised Black Black-Colored Lesion with Irregular Borders without Surface Ulceration Located in the Right Temporal Area of the Face. and prominent nucleoli infiltrated the junctional area of the epidermis and dermis, as well as in the upper part of the dermis and papillary dermis. Melanin pigment was found in both epidermal cells and upper dermis. There was no invasion of malignant cells into the deeper parts of the dermis. A number of malignant melanocytic cells were evident within the vascular spaces of the upper dermis (Figure 4).

\section{Discussion}

Malignant melanoma is a malignancy of melanocytic cells that could affect the skin, eyes, mucosal membranes, anus, and leptomeningeal tissue. ${ }^{1,2}$ Melanoma can arise on a pre-existing nevus. Malignant melanoma originates from melanocytic cells in the skin or retina of the eyes or mucous membranes. ${ }^{3}$ Wounding of a previous mole or sudden enlargement of a pre-existing mole or discoloration can be a clue to indicate the malignant transformation of this type of mole. ${ }^{4}$ People with fair skin and long-term exposure to the sun are more likely to develop malignant melanoma. Lentigo maligna melanoma is a subtype of malignant melanoma that mostly affects the head and neck skin tissue; it could behave aggressively and metastasize to other organs. Regional lymph nodes are involved in the early phase of metastasis and then neoplastic melanocytic cells could spread via vascular channels and involve internal organs such as the liver, lung, brain, and GI tract. ${ }^{5}$ Metastasis to the appendix without the involvement of regional lymph nodes is very rare, and all of the cases reported have had a previous history of malignant melanoma, whereas our patient was not a known case of malignant melanoma and had not received any treatment. ${ }^{1-8}$ In the differential diagnosis of cancers involving the appendix, neuroendocrine tumors are at the top of the list, as well as primary appendiceal cancers such as mucinous carcinoma, adenocarcinoma, and also malignant lymphoma. ${ }^{6}$ One of the notable points in this case was the nuclear morphology of the tumor cells,

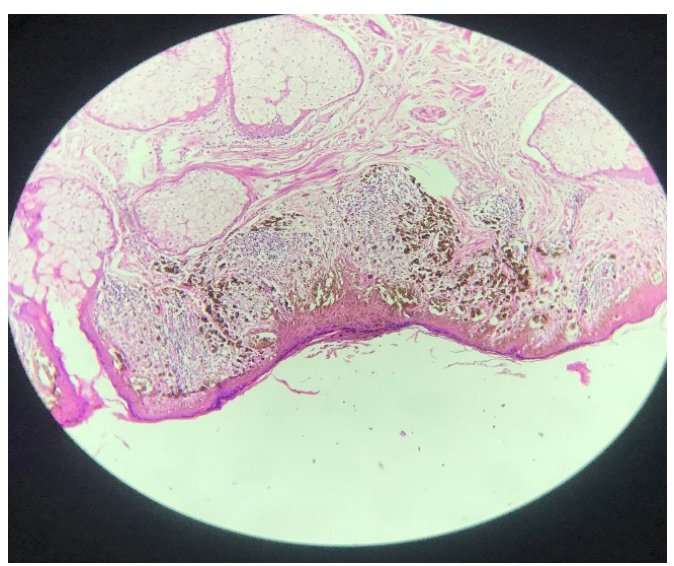

Figure 4. Neoplastic Melanocytes in the Dermis and Epidermis with Superficially Intravascular Invasion (H\&E staining, $\times 20$ ). 
which had irregular hyperchromic nuclei without distinct eosinophilic nucleoli. Tumor cells were arranged in solid clusters and gland-like structures. Neoplastic cells in a neuroendocrine tumor have round nuclei with a pepperand-salt chromatin pattern and are arranged in solid nests; in IHC studies, they have a positive reaction pattern for chromogranin A, NSE, synaptophysin and CD56. Neoplastic cells of mucinous adenocarcinoma have large nuclei and abundant mucin filled cytoplasm; however, poorly differentiated cells lack mucinous cytoplasm and are arranged in nests and a single-cell pattern. Neoplastic melanocytes have large nuclei and prominent nucleoli with melanin pigment in the cytoplasm of tumoral cells; however, in our case, the neoplastic cells were small to medium size and had an irregular nuclear contour, and rarely had the melanin pigment. Neoplastic melanocytes have a positive reaction pattern for S-100, HMB-45, and Melan A. In previous studies, all patients had a previous history of treated malignant melanoma and differed from our case who had not been previously diagnosed with malignant melanoma. ${ }^{9,10}$ Another interesting finding in our patient was that the regional and non-regional lymph nodes were not involved. Our patient received immunotherapy and target therapy after surgical resection of the face skin lesion, and after 3 years, he had multiple organ metastases.

\section{Authors' Contribution}

STZ: Case report design, final editing, pathology reports. KR: Data collection, manuscript editing, review of literatures. SAH: Data collection, references review, final edinting.

\section{Conflict of Interest Disclosures}

The authors declare that they have no conflict of interest.

\section{Ethical Statement}

The consent form was signed by the patient.

\section{References}

1. Schuchter LM, Green R, Fraker D. Primary and metastatic diseases in malignant melanoma of the gastrointestinal tract. Curr Opin Oncol. 2000;12(2):181-5. doi: 10.1097/00001622-200003000-00014.

2. Patel JK, Didolkar MS, Pickren JW, Moore RH. Metastatic pattern of malignant melanoma. A study of 216 autopsy cases. Am J Surg. 1978;135(6):807-10. doi: 10.1016/00029610(78)90171-x.

3. Liang KV, Sanderson SO, Nowakowski GS, Arora AS. Metastatic malignant melanoma of the gastrointestinal tract. Mayo Clin Proc. 2006;81(4):511-6. doi:10.4065/81.4.511.

4. DasGupta T, Brasfield R. Metastatic melanoma. A clinicopathological study. Cancer. 1964;17:132339. doi:10.1002/1097-0142(196410)17:10<1323::aidcncr2820171015>3.0.co;2-n.

5. Akaraviputh $\mathrm{T}$, Arunakul S, Lohsiriwat $\mathrm{V}$, Iramaneerat $\mathrm{C}$, Trakarnsanga A. Surgery for gastrointestinal malignant melanoma: experience from surgical training center. World J Gastroenterol. 2010;16(6):745-8. doi: 10.3748/wjg.v16. i6.745.

6. Elsayed AM, Albahra M, Nzeako UC, Sobin LH. Malignant melanomas in the small intestine: a study of 103 patients. Am J Gastroenterol. 1996;91(5):1001-6.

7. Blecker D, Abraham S, Furth EE, Kochman ML. Melanoma in the gastrointestinal tract. Am J Gastroenterol. 1999;94(12):342733. doi: 10.1111/j.1572-0241.1999.01604.x.

8. Letovanec I, Vionnet $\mathrm{M}$, Bouzourene $\mathrm{H}$. Primary appendiceal melanoma: fiction or reality?. Hum Pathol. 2004;35(5):627-9. doi: 10.1016/j.humpath.2003.10.028.

9. Alduaij AA, Resnick MB, Kawata M, Pricolo VE. Metastatic malignant melanoma presenting as an appendiceal mucocele. J Oncol. 2011;2011:546570. doi: 10.1155/2011/546570.

10. Kitano M, Maker AV, Lanier BJ, Danforth DN, Kammula US. Appendicitis secondary to metastatic melanoma: review of the National Institutes of Health experience. JAMA Surg. 2014;149(7):735-8. doi: 10.1001/jamasurg.2013.4067. 